\title{
Pigmentos à base de cobalto para aplicação em revestimentos cerâmicos
}

\section{(Cobalt-based pigments for application in ceramic coatings)}

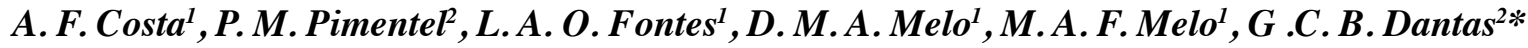 \\ ${ }^{1}$ Universidade Federal do Rio Grande do Norte, Laboratório de Tecnologia Ambiental, \\ Av. Sen. Salgado Filho 3000, 59078-970, Natal, RN, Brasil \\ ${ }^{2}$ Universidade Federal Rural do Semi-Árido, 59515-000, Angicos, RN, Brasil
}

\begin{abstract}
Resumo
Pigmentos inorgânicos foram preparados com estrutura espinélio para aplicações em revestimentos cerâmicos. Pigmentos com composição $\mathrm{CoCr}_{2} \mathrm{O}_{4}$ e $\mathrm{Co}_{0,8} \mathrm{Zn}_{0,2} \mathrm{Cr}_{2} \mathrm{O}_{4}$ foram sintetizados por uma rota química usando gelatina de uso farmacêutico como precursor orgânico. Foram investigadas a influência da dopagem e a temperatura de tratamento térmico na coloração dos pigmentos e dos esmaltes cerâmicos. Os materiais foram caracterizados por análise termogravimétrica, difração de raios X, microscopia eletrônica de varredura, espectroscopia por energia dispersiva, espectroscopia na região do UV-visível e colorimetria. Os pós apresentaram estrutura espinélio a partir de $500{ }^{\circ} \mathrm{C}$, com formação de fase secundária residual de $\mathrm{Cr}_{2} \mathrm{O}_{3}$ nas amostras $\mathrm{Co}_{0.8} \mathrm{Zn}_{0.2} \mathrm{Cr}_{2} \mathrm{O}_{4} \operatorname{tratadas}$ termicamente a 500 e $1000{ }^{\circ} \mathrm{C}$. Os pigmentos e os esmaltes preparados apresentaram cores em tons de azul esverdeado para as amostras $\mathrm{CoCr}_{2} \mathrm{O}_{4}$ e verde para a amostra $\mathrm{Co}_{0,8} \mathrm{Zn}_{0,2} \mathrm{Cr}_{2} \mathrm{O}_{4}$. Os resultados confirmaram a efetividade da rota de síntese utilizada para produzir óxidos com estrutura espinélio.

Palavras-chave: síntese de espinélios, pigmentos cerâmicos, gelatina.
\end{abstract}

\begin{abstract}
Inorganic pigments were prepared with spinel structure for applications in ceramic tiles. Pigments with composition $\mathrm{CoCr}_{2} \mathrm{O}_{4}$ and $\mathrm{Co}_{0.8} \mathrm{Zn}_{0.2} \mathrm{Cr}_{2} \mathrm{O}_{4}$ were synthesized by a chemical route using gelatin for pharmaceutical use as an organic precursor. The influences of doping and thermal treatment temperature on pigment and ceramic glaze color were investigated. The materials were characterized by thermogravimetric analysis, $X$-ray diffraction, scanning electron microscopy, dispersive energy spectroscopy, spectroscopy in the UV-visible region and colorimetry. The powders treated at $500{ }^{\circ} \mathrm{C}$ presented spinel structure, with the formation of residual secondary phase of $\mathrm{Cr}_{2} \mathrm{O}_{3}$ in samples $\mathrm{Co}_{0.8} \mathrm{Zn}_{0.2} \mathrm{Cr}_{2} \mathrm{O}_{4}$ heat-treated at 500 and $1000{ }^{\circ} \mathrm{C}$. The prepared pigments and glazes presented blue-green colors for the samples $\mathrm{CoCr} \mathrm{O}_{4}$ and green color for the sample $\mathrm{Co}_{0.8} \mathrm{Zn}_{0.2} \mathrm{Cr}_{2} \mathrm{O}_{4}$. The results confirmed the effectiveness of the synthesis route used to produce oxides with a spinel structure.
\end{abstract}

Keywords: synthesis of spinel, ceramic pigments, gelatin.

\section{INTRODUÇÃO}

Os espinélios representam uma das estruturas cristalinas mais interessantes na produção de pigmentos estáveis e quimicamente inertes. Os íons de diferentes estados de oxidação estão presentes tanto em sítios tetraédricos quanto em octaédricos, podendo gerar óxidos com estrutura espinélio normal ou invertido. Recentemente os óxidos com estrutura espinélio baseados em cromitas vêm se destacando devido às suas propriedades elétricas, com aplicações abrangendo vários campos da física, ciências de materiais e geofísica. Nas cromitas com estrutura espinélio de formulação química $\mathrm{ACr}_{2} \mathrm{O}_{4}$, o sítio A é composto por um metal com estado de oxidação $2+$ e desempenha $o$ papel de modificador da rede, posicionando-se nos sítios tetraédricos. Já o cromo desempenha o papel de formador de rede, localizado nos sítios octaédricos. Modificações nas

*gerbeson_dantas@hotmail.com

Dhttps://orcid.org/0000-0002-6662-5789 propriedades dos diversos tipos de espinélios formados podem acontecer devido à variação do tipo de íon A [1]. A possibilidade de acomodação de metais de transição com estado de oxidação 2+ no sítio A da estrutura de espinélios baseados em cromitas conduzem à formação de diferentes cores, sendo estas estáveis e intensas, adequadas ao uso como pigmentos [2]. Desta forma, é possível atender à demanda crescente da indústria cerâmica por pigmentos nas diversas cores, sobretudo azuis, verdes, amarelos e vermelhos [3].

Para fazer a seleção de pigmentos cerâmicos nas mais diversas aplicações, devem ser considerados alguns fatores e características, tais como: insolubilidade na cobertura, resistência ao ataque por agentes químicos (ácidos e bases), estabilidade térmica, distribuição granulométrica homogênea e ausência de emissões gasosas no interior dos vidrados, que provocam defeitos nos mesmos [4-6]. Geralmente a indústria cerâmica brasileira obtém pigmentos por meio da síntese de óxidos mistos pelo método convencional [7]. Entretanto, 
muitos são os esforços no sentido de produzir pigmentos de maior qualidade e melhor desempenho, minimizando os danos ambientais provocados pela sua obtenção. Assim, uma das soluções frequentemente estudadas é o desenvolvimento de novas rotas de síntese que substituam o método convencional, pois este resulta em pigmentos com propriedades heterogêneas e o processo envolve um alto gasto de energia $[8,9]$.

Nos últimos anos, tem sido proposta uma rota alternativa baseada na utilização da gelatina como precursor orgânico [10-12]. A rota gelatina consiste em formar dispersões coloidais entre os íons metálicos com grupos coordenantes da gelatina, tais como amina $\left(\mathrm{NH}_{2}\right)$ e o grupo carboxílico $(\mathrm{COOH})$, que são submetidos a aquecimento para redução do volume até a formação de um gel e, em seguida, tratados termicamente a uma temperatura pré-determinada para tornar-se um óxido inorgânico [13]. Sua principal vantagem é a não geração de gases, ser atóxico e não necessitar da formação do sol, reduzindo substancialmente o tempo de síntese $[10,12]$. A gelatina possui uma grande capacidade de biodegradabilidade e biocompatibilidade em ambientes fisiológicos, fazendo com que a utilização desse produto em algumas sínteses seja de amplo interesse científico e tecnológico [14]. No presente estudo foram sintetizados pigmentos cerâmicos, com estrutura de espinélio, de composição $\mathrm{CoCr}_{2} \mathrm{O}_{4}$ e $\mathrm{Co}_{0,8} \mathrm{Zn}_{0,2} \mathrm{Cr}_{2} \mathrm{O}_{4}$ utilizando gelatina como precursor orgânico. Os pigmentos foram incorporados em esmalte transparente brilhante para avaliar o desenvolvimento da cor e a estabilidade da mesma, após tratamento térmico a 500,800 e $1000^{\circ} \mathrm{C}$.

\section{MATERIAIS E MÉTODOS}

Sintese dos pigmentos: para a síntese dos compostos $\mathrm{CoCr}_{2} \mathrm{O}_{4}$ e $\mathrm{Co}_{0,8} \mathrm{Zn}_{0,2} \mathrm{Cr}_{2} \mathrm{O}_{4}\left(\mathrm{Co}_{\mathrm{x}} \mathrm{Zn}_{1-\mathrm{x}} \mathrm{Cr}_{2} \mathrm{O}_{4}\right)$ foram utilizados o nitrato de zinco hexa-hidratado $\left[\mathrm{Zn}\left(\mathrm{NO}_{3}\right)_{2} \cdot 6 \mathrm{H}_{2} \mathrm{O}\right.$, Vetec, $98 \%$ ], nitrato de cobalto hexa-hidratado $\left[\mathrm{Co}\left(\mathrm{NO}_{3}\right)_{2} \cdot 6 \mathrm{H}_{2} \mathrm{O}\right.$, Acros Organics, 99\%] e o nitrato de cromo nonahidratado $\left[\mathrm{Cr}\left(\mathrm{NO}_{3}\right)_{3} \cdot 9 \mathrm{H}_{2} \mathrm{O}\right.$, Vetec, 97\%] em quantidades estequiométricas. Foi utilizada a razão massa/massa 1:1 de gelatina e cátions metálicos. Para a preparação do composto $\mathrm{CoCr}_{2} \mathrm{O}_{4}$ foi utilizado o método gelatina [1012]. Inicialmente foi adicionada a gelatina em um béquer com $150 \mathrm{~mL}$ de água deionizada sob agitação constante a $50{ }^{\circ} \mathrm{C}$ para dissolvê-la. A dispersão permaneceu sob aquecimento até aproximadamente $70{ }^{\circ} \mathrm{C}$. Em seguida foi acrescentado o nitrato de cobalto sob agitação por $15 \mathrm{~min}$ e, posteriormente, foi acrescentado o nitrato de cromo, mantendo a agitação por $60 \mathrm{~min}$. Após esse tempo, a temperatura foi aumentada para $90{ }^{\circ} \mathrm{C}$ ocorrendo assim a evaporação da água e formação de uma resina polimérica, que foi tratada termicamente a $350{ }^{\circ} \mathrm{C}$, com patamar de 2 h e taxa de aquecimento de $10{ }^{\circ} \mathrm{C} / \mathrm{min}$ para a redução da matéria orgânica. $\mathrm{O}$ mesmo procedimento foi adotado para o composto $\mathrm{Co}_{\mathrm{x}} \mathrm{Zn}_{1-\mathrm{x}} \mathrm{Cr}_{2} \mathrm{O}_{4}$. Os pós precursores resultantes deste processo foram tratados termicamente utilizando ciclo térmico com aquecimento a $10{ }^{\circ} \mathrm{C} / \mathrm{min}$ e patamar de
$4 \mathrm{~h}$ nas temperaturas de 500,800 ou $1000{ }^{\circ} \mathrm{C}$ para obtenção da fase.

Aplicação no esmalte: foi utilizado um esmalte cerâmico transparente brilhante com composição percentual em massa de $80 \%$ de feldspato e $20 \%$ de bórax $\left(\mathrm{Na}_{2} \mathrm{~B}_{4} \mathrm{O}_{7} \cdot 10 \mathrm{H}_{2} \mathrm{O}\right.$, Vetec, 99,5\%). Os revestimentos foram obtidos usando misturas de $2 \%$ em massa de pigmento para 98\% numa massa de esmalte contendo uma concentração mássica de $0,2 \%$ de carboximetilcelulose (CMC, Synth, $99,5 \%$ ) e $0,1 \%$ de hexametafosfato de sódio. A barbotina resultante foi aplicada à base cerâmica, que foi levada à mufla e submetida ao tratamento térmico a $1100{ }^{\circ} \mathrm{C}$, com taxa de aquecimento de $15^{\circ} \mathrm{C} / \mathrm{min}$ e patamar de $15 \mathrm{~min}$.

Caracterização: as fases cristalinas dos pós tratados termicamente a 500,800 e $1000{ }^{\circ} \mathrm{C}$ foram identificadas por difração de raios $\mathrm{X}$ (DRX). O equipamento utilizado (XRD-6000, Shimadzu) foi operado com radiação $\mathrm{CuK \alpha}$. Os difratogramas foram obtidos com ângulo $2 \theta$ variando de $10^{\circ}$ a $80^{\circ}$. Os padrões de difração foram ajustados e refinados pelo método Rietveld [15] e o ajuste do padrão foi feito com o software MAUD [16]. A análise termogravimétrica (TG) foi realizada por meio do equipamento STA 449 F3 Júpiter, Netzsch, em atmosfera de ar, com taxa de aquecimento de $10{ }^{\circ} \mathrm{C} / \mathrm{min}$. As micrografias foram obtidas por meio do microscópio eletrônico de varredura (MEV, XL-30ESEM, Philips) com tensão de $20 \mathrm{kV}$. As medições colorimétricas do CIE-L*a*b* dos pós e das cerâmicas foram realizadas usando um colorímetro (Color-Eye 2180, Gretag Macbeth). Os espectros de reflectância foram obtidos em um espectrofotômetro (UV-Visible, Shimadzu) equipado com reflectância acessória UV-2550, com comprimento de onda na região de 200-800 nm.

\section{RESULTADOS E DISCUSSÃO}

As curvas termogravimétricas dos pós precursores são mostradas na Fig. 1. Observou-se uma perda de massa contínua entre a temperatura ambiente e $900{ }^{\circ} \mathrm{C}$, atribuída à eliminação de água adsorvida e à decomposição dos aminoácidos presentes na estrutura orgânica da gelatina [17]. As amostras apresentaram pequenas quantidades de perda de massa, sendo $5,3 \%$ e $5 \%$ para os pós $\mathrm{CoCr}_{2} \mathrm{O}_{4}$ e $\mathrm{Co}_{\mathrm{x}} \mathrm{Zn}_{1-\mathrm{x}} \mathrm{Cr}_{2} \mathrm{O}_{4}$, respectivamente. A perda de massa mais acentuada só ocorreu a partir de $700{ }^{\circ} \mathrm{C}$. Este comportamento ocorreu devido às fortes interações ácidobase de Lewis entre os grupos funcionais da gelatina (bases de Lewis) e os elementos metálicos (ácidos de Lewis) [17].

A Fig. 2 apresenta os difratogramas de raios $\mathrm{X}$ padrão e experimental dos compostos $\mathrm{CoCr}_{2} \mathrm{O}_{4}$ e $\mathrm{Co}_{\mathrm{x}} \mathrm{Zn}_{1-\mathrm{x}} \mathrm{Cr}_{2} \mathrm{O}_{4}$ tratados a 500,800 e $1000{ }^{\circ} \mathrm{C}$. No composto $\mathrm{CoCr}_{2} \mathrm{O}_{4}$, em todas essas temperaturas, foram identificados picos de reflexão característicos da estrutura espinélio tipo cúbica, de acordo com a ficha JCPDS 80-1668. Não foram obeservadas reflexões atribuídas a outras fases dentro do limite de detecção da técnica de DRX. Este comportamento ocorreu para os materiais obtidos por tratamento térmico realizados nas três temperaturas. À medida que a 

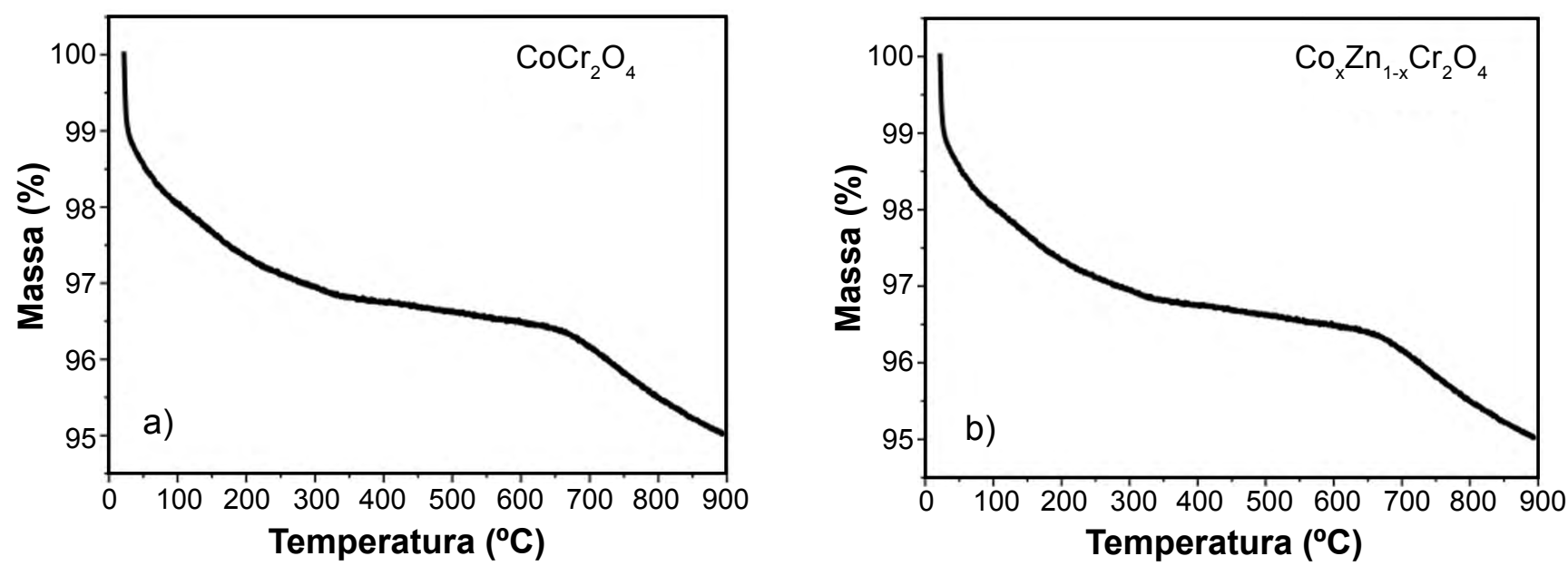

Figura 1: Curvas de análise termogravimétrica (TG) dos pós precursores: a) $\mathrm{CoCr}_{2} \mathrm{O}_{4}$; e b) $\mathrm{Co}_{\mathrm{x}} \mathrm{Zn}_{1-\mathrm{x}} \mathrm{Cr}_{2} \mathrm{O}_{4}$.

[Figure 1: Thermogravimetric analysis (TG) curves of precursor powders: a) $\mathrm{CoCr}_{2} \mathrm{O}_{4}$; and b) $\mathrm{Co}_{x} \mathrm{Zn}_{1-x} \mathrm{Cr}_{2} \mathrm{O}_{4}$.]

temperatura do tratamento térmico das amostras aumentou, os picos tornaram-se mais estreitos e definidos, o que pode ser atribuído a um aumento na cristalinidade destes compostos com a elevação da temperatura de tratamento térmico, indicando que o material foi se organizando a longo alcance. $\mathrm{O}$ composto $\mathrm{Co}_{\mathrm{x}} \mathrm{Zn}_{1-\mathrm{x}} \mathrm{Cr}_{2} \mathrm{O}_{4}$ apresentou simetria cúbica, conforme ficha JCPDS 82-2251. Estes resultados evidenciaram que o método de síntese foi efetivo na obtenção da fase espinélio quando os pós precursores foram submetidos a tempo e temperatura de processamento relativamente baixo como $500{ }^{\circ} \mathrm{C}$, o que é uma das vantagens quando comparado com outras rotas de síntese, nas quais a fase espinélio é obtida em temperaturas mais altas. Eliziário [18] preparou pigmentos com estrutura $\mathrm{CoCr}_{2} \mathrm{O}_{4}$ pelo método dos precursores poliméricos, obtendo a fase majoritária a partir de $600{ }^{\circ} \mathrm{C}$. Gonçalves et al. [19] inseriram ferro na estrutura dessa cromita usando o método Pechini, mas não obtiveram pós monofásicos, mesmo com aquecimento a $1000{ }^{\circ} \mathrm{C}$. Betancur-Granados e Restrepo-
Baena [20] sintetizaram espinélios de $\mathrm{CoCr}_{2} \mathrm{O}_{4}$ pelo método convencional, mas só obtiveram a fase quando trataram termicamente a $1200{ }^{\circ} \mathrm{C} / 6$ h. Já Tian et al. [21] obtiveram a fase pelo método hidrotérmico, entretanto somente após $24 \mathrm{~h}$ de tratamento, enquanto Coelho et al. [22] prepararam pigmentos cerâmicos à base de cromita pelo método de reação de combustão, todavia o início da formação da estrutura espinélio só foi observada a partir de $600{ }^{\circ} \mathrm{C}$, mas com presença de fase secundária. Logo, o método gelatina mostrou-se vantajoso na obtenção de cromitas com estrutura espinélio, considerando que a fase já foi obtida a $500{ }^{\circ} \mathrm{C}$, enquanto outros estudos, mesmo usando rotas mais demoradas e temperaturas de tratamento térmico superiores, não conseguiram resultados similares. Nas amostras sintetizadas a 500 e $1000{ }^{\circ} \mathrm{C}$ foram observadas reflexões residuais atribuídas à fase $\mathrm{Cr}_{2} \mathrm{O}_{3}$ em frações de $1,2 \%$ a $500{ }^{\circ} \mathrm{C}$ e $2,2 \%$ a $1000{ }^{\circ} \mathrm{C}$ (Tabela I). Foi determinado, em estudos com espinélios baseados em cromitas [19, 23-25], que em baixas temperaturas
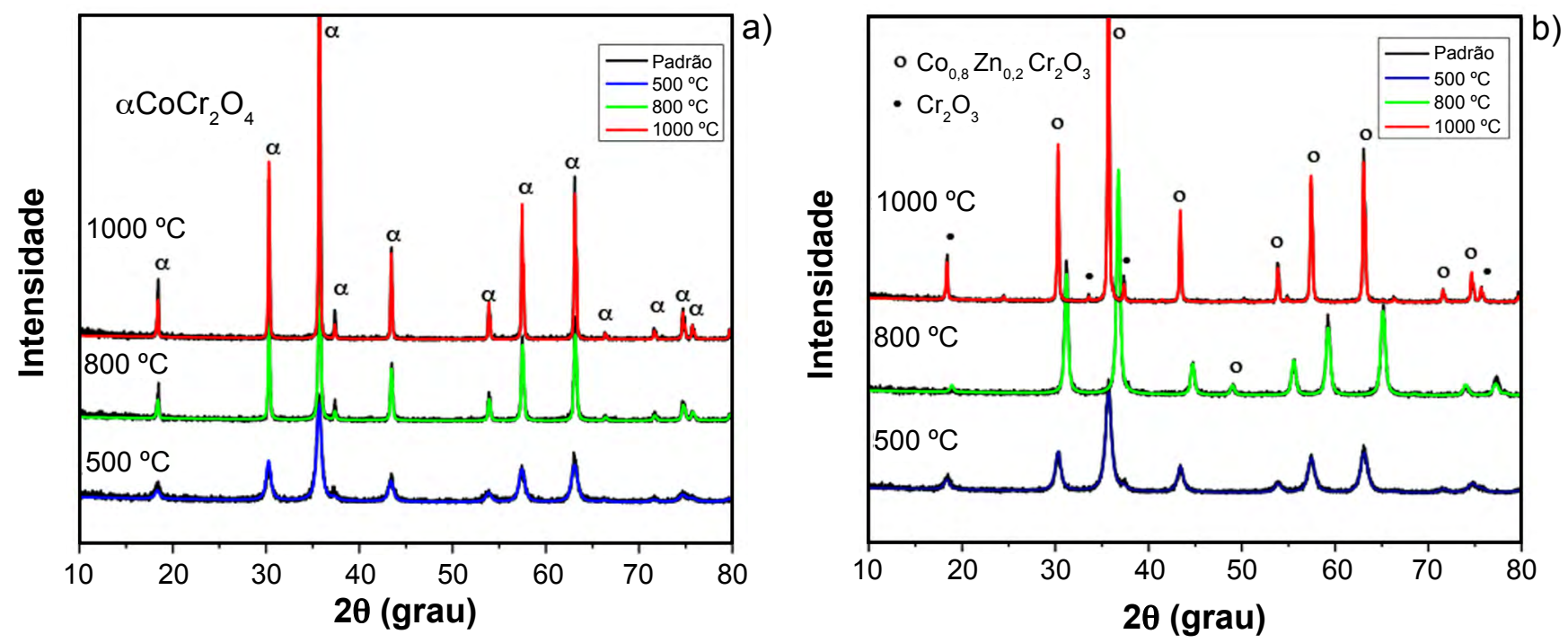

Figura 2: Difratogramas de raios X padrão e experimental das amostras: a) $\mathrm{CoCr}_{2} \mathrm{O}_{4}$; e b) $\mathrm{Co}_{\mathrm{x}} \mathrm{Zn}_{1-\mathrm{x}} \mathrm{Cr}_{2} \mathrm{O}_{4}$. [Figure 2: Standard and experimental $\mathrm{X}$-ray diffraction patterns of the samples: a) $\mathrm{CoCr}_{2} \mathrm{O}_{4}$; and b) $\mathrm{Co}_{x} \mathrm{Zn}_{1-x} \mathrm{Cr}_{2} \mathrm{O}_{4} \cdot$ ] 
Tabela I - Parâmetros microestruturais dos compostos $\mathrm{CoCr}_{2} \mathrm{O}_{4}$ e $\mathrm{Co}_{\mathrm{x}} \mathrm{Zn}_{1-\mathrm{x}} \mathrm{Cr}_{2} \mathrm{O}_{4}$ refinados por Rietveld. [Table I - Microstructural parameters of the $\mathrm{CoCr}_{2} \mathrm{O}_{4}$ and $\mathrm{Co}_{2} \mathrm{Zn}_{1-x} \mathrm{Cr}_{2} \mathrm{O}_{4}$ compounds refined by Rietveld.]

\begin{tabular}{cccccccccc}
\hline Composto & $\begin{array}{c}\mathrm{T} \\
\left({ }^{\circ} \mathrm{C}\right)\end{array}$ & $\mathrm{SIG}$ & $\mathrm{Rw}$ & $\begin{array}{c}\text { Espinélio } \\
(\% \text { massa) }\end{array}$ & $\begin{array}{c}\mathrm{Cr}_{2} \mathrm{O}_{3} \\
(\% \text { massa })\end{array}$ & $\begin{array}{c}\mathrm{a} \\
(\AA)\end{array}$ & $\begin{array}{c}\mathrm{Dc} \\
(\mathrm{nm})\end{array}$ & $\begin{array}{c}\mathrm{Vc} \\
\left(\AA^{3}\right)\end{array}$ & $\begin{array}{c}\text { Cristalinidade } \\
(\%)\end{array}$ \\
\hline \multirow{2}{*}{$\mathrm{CoCr}_{2} \mathrm{O}_{4}$} & 500 & 1,16 & 29,00 & 100 & - & 8,33 & 21,36 & 578,0 & 59,18 \\
& 800 & 1,16 & 29,52 & 100 & - & 8,32 & 99,24 & 575,9 & 92,37 \\
& 1000 & 1,20 & 30,00 & 100 & - & 8,32 & 270,0 & 575,9 & 98,38 \\
\hline \multirow{2}{*}{$\mathrm{Co}_{\mathrm{x}} \mathrm{Zn}_{1-\mathrm{x}} \mathrm{Cr}_{2} \mathrm{O}_{4}$} & 500 & 1,20 & 19,04 & 98,8 & 1,2 & 8,33 & 16,77 & 578,0 & 59,18 \\
& 1000 & 1,18 & 15,65 & 100 & - & 8,09 & 35,10 & 529,5 & 71,00 \\
\hline
\end{tabular}

$T$ - temperatura de tratamento; SIG, Rw - parâmetros de ajuste do refinamento; a - parâmetro de rede; Dc - tamanho de cristalito; Vc - volume de célula.

estes compostos são relativamente estáveis, mas em temperaturas superiores a $900{ }^{\circ} \mathrm{C}$ apresentam tendência de decomposição e formação de fases $\mathrm{Cr}_{2} \mathrm{O}_{3}$ e $\mathrm{ACrO}_{2}$.

A Fig. 3a ilustra a variação da cristalinidade e tamanho do cristalito do $\mathrm{CoCr}_{2} \mathrm{O}_{4}$ nas diferentes temperaturas. Observou-se que, à medida que aumentou a temperatura de tratamento térmico, ocorreu um aumento da cristalinidade. Isto ocorreu porque a elevação da temperatura favoreceu uma maior acomodação dos átomos na estrutura cristalina. O pó tratado a $1000{ }^{\circ} \mathrm{C}$ apresentou o maior grau de cristalinidade ( $>98 \%$ ), enquanto os pós tratados a $500 \mathrm{e}$ $800{ }^{\circ} \mathrm{C}$ apresentaram $59,18 \%$ e $92,37 \%$, respectivamente. O mesmo ocorreu no tamanho dos cristalitos (Dc), onde se pôde verificar que com a elevação da temperatura ocorreu um aumento do tamanho. O parâmetro de rede (a) e o volume de célula $(\mathrm{Vc})$ dos pós puro e dopado estão mostrados na Tabela I. Os valores do parâmetro de rede são indicativos de espinélio com simetria cúbica variando de 8,09 a $8,33 \AA$ nos pós sintetizados. Os valores do volume de célula variaram de 529,5 a $578,9 \AA^{3}$. A Fig. $3 b$ ilustra a variação da cristalinidade e tamanho do cristalito para o composto $\mathrm{Co}_{\mathrm{x}} \mathrm{Zn}_{1-\mathrm{x}} \mathrm{Cr}_{2} \mathrm{O}_{4}$. Observou-se que com o aumento da temperatura de tratamento ocorreu uma elevação gradual da cristalinidade. Isto pôde ser verificado observando-se o claro estreitamento da reflexão de Bragg em aproximadamente $37^{\circ}$, que é indicativo do aumento do grau de cristalinidade das amostras e, consequentemente, provocou um aumento do tamanho de cristalito para este pigmento. A $1000{ }^{\circ} \mathrm{C}$ o valor para o tamanho médio de cristalito foi em torno de $135 \mathrm{~nm}$ e a cristalinidade de 93,22\%. O parâmetro de rede para essa mesma temperatura foi de $8,33 \AA$ e o volume de célula de $578,9 \AA^{3}$. Os valores de otimização do refinamento (SIG) foram aceitáveis (inferior a 2) para ambas amostras, característico de um bom refinamento [17]. As micrografias de MEV podem ser observadas na Fig. 4. Ficou evidenciada morfologia com formação de aglomerados com formatos variados e contornos arredondados de diferentes tamanhos nos compostos $\mathrm{CoCr}_{2} \mathrm{O}_{4}$ e $\mathrm{Co}_{\mathrm{x}} \mathrm{Zn}_{1-\mathrm{x}} \mathrm{Cr}_{2} \mathrm{O}_{4}$.

Os espectros de refletância na região do UV-vis dos pigmentos de $\mathrm{CoCr}_{2} \mathrm{O}_{4}$ e $\mathrm{Co}_{\mathrm{x}} \mathrm{Zn}_{1-\mathrm{x}} \mathrm{Cr}_{2} \mathrm{O}_{4}$ são apresentados na Fig. 5. A primeira banda entre 200 e $300 \mathrm{~nm}$ foi relacionada à transferência de carga e não influenciou na cor, pois ocorreu fora da região do visível. Essa banda foi ficando mais intensa à medida que se aumentou a temperatura. Uma segunda banda mais estreita em torno de $380 \mathrm{~nm}$, com pouca intensidade na região do violeta, foi ficando mais definida com o aumento da temperatura, resultado da transferência de carga de alta energia entre Co e O [26]. Uma terceira banda bastante intensa foi observada próximo a $530 \mathrm{~nm}$,
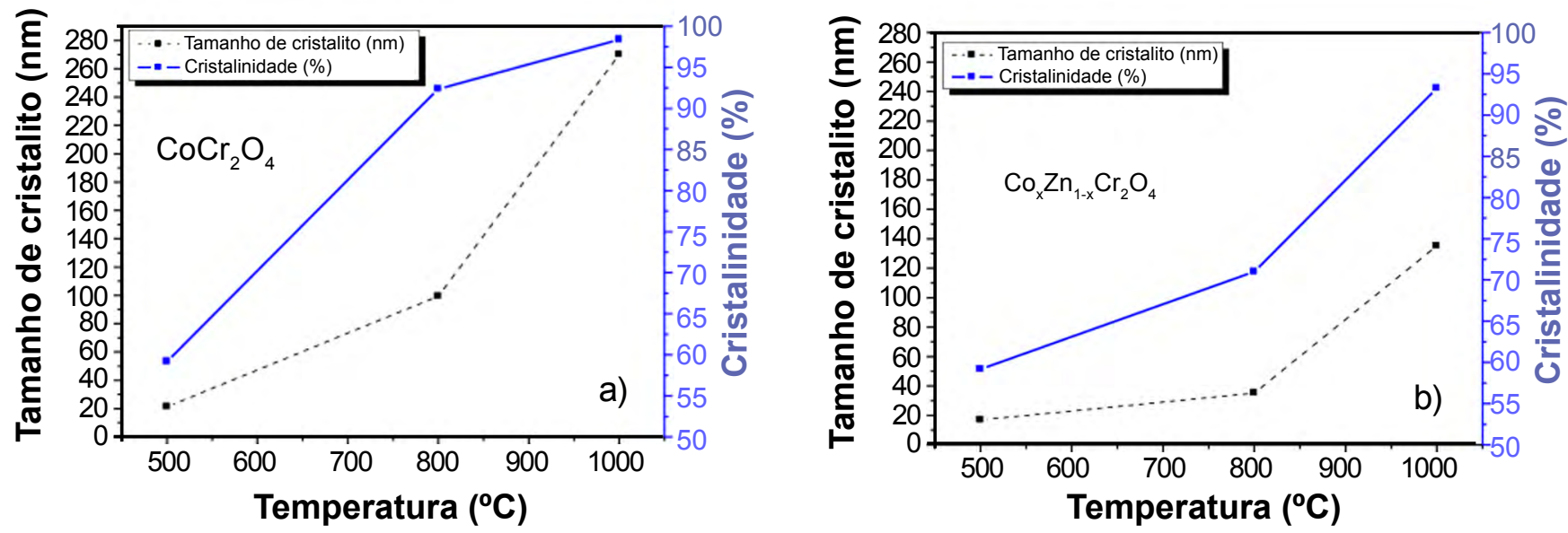

Figura 3: Tamanho médio de cristalito e cristalinidade em função da temperatura para: a) $\mathrm{CoCr}_{2} \mathrm{O}_{4}$; e b) $\mathrm{Co}_{\mathrm{x}} \mathrm{Zn}_{1-\mathrm{x}} \mathrm{Cr}_{2} \mathrm{O}_{4}$. [Figure 3: Average crystallite size and crystallinity as a function of temperature for: a) $\mathrm{CoCr}_{2} \mathrm{O}_{4}$; and b) $\mathrm{Co}_{x} \mathrm{Zn}_{1-x} \mathrm{Cr}_{2} \mathrm{O}_{4}$.] 

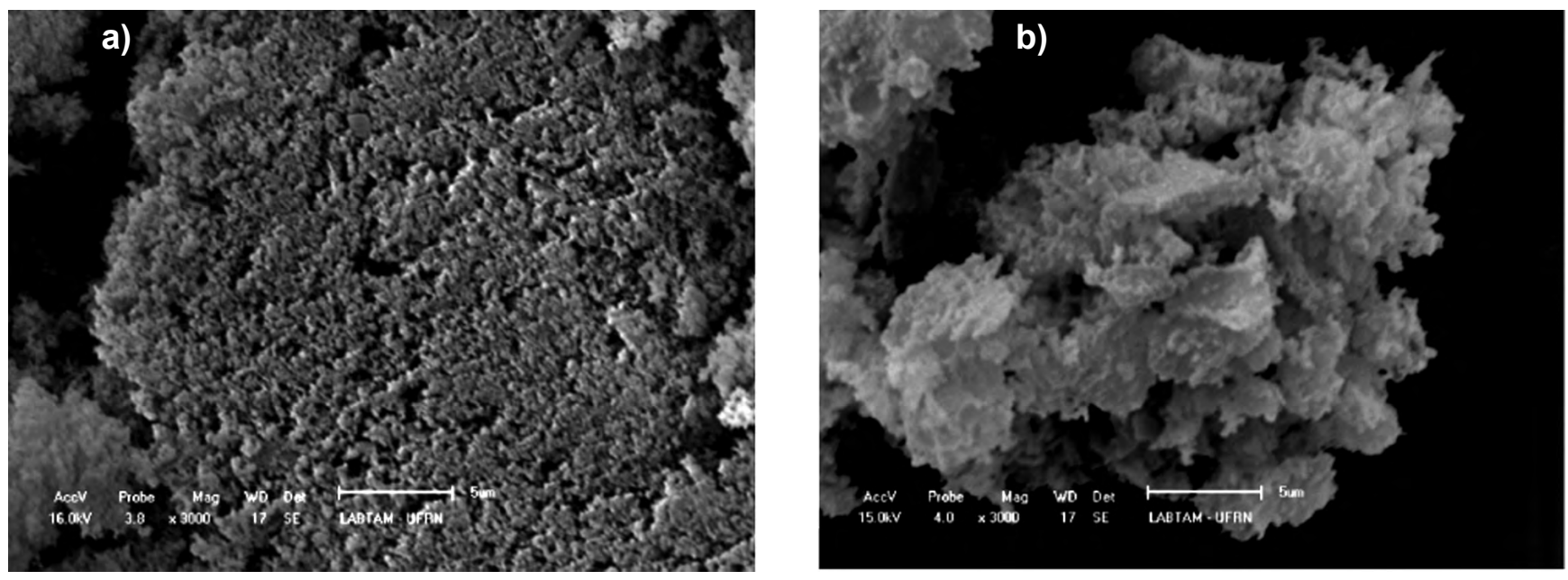

Figura 4: Micrografias de MEV dos pigmentos $\mathrm{CoCr}_{2} \mathrm{O}_{4}$ (a) e $\mathrm{Co}_{\mathrm{x}} \mathrm{Zn}_{1-\mathrm{x}} \mathrm{Cr}_{2} \mathrm{O}_{4}$ (b) tratados termicamente a $800{ }^{\circ} \mathrm{C}$. [Figure 4: SEM micrographs of the pigments $\mathrm{CoCr}_{2} \mathrm{O}_{4}\left(\right.$ a) and $\mathrm{Co}_{x} \mathrm{Zn}_{1-x} \mathrm{Cr}_{2} \mathrm{O}_{4}(\mathrm{~b})$ heat treated at $800{ }^{\circ} \mathrm{C}$.]

atribuída à transição do $\mathrm{Cr}^{3+}$. A cor deste composto foi resultado da sobreposição das bandas dos íons $\mathrm{Co}^{2+} \mathrm{e} \mathrm{Cr}^{3+}$, originando uma cor azul esverdeada. Segundo Souza et al. [27], este comportamento é esperado, pois geralmente o $\mathrm{Co}^{2+}$ em ambiente tetraédrico e $\mathrm{Cr}^{3+} \mathrm{em}$ ambiente octaédrico decorre nas cores azul e verde, respectivamente. Observouse também que os espectros de reflectância obtidos para o material dopado nas temperaturas de tratamento de 500, 800 e $1000{ }^{\circ} \mathrm{C}$ apresentaram similaridade com os espectros de reflectância obtidos para a amostra $\mathrm{CoCr}_{2} \mathrm{O}_{4}$. O íon $\mathrm{Cr}^{3+}$ quando em sítio octaédrico apresenta quatro transições possíveis na região do visível: a primeira transição e mais pronunciada ocorre em cerca de 530-570 nm e é atribuída a ${ }^{4} \mathrm{~A}_{2 \mathrm{~g}}\left({ }^{4} \mathrm{~F}\right) \rightarrow{ }^{4} \mathrm{~T}_{2 \mathrm{~g}}\left({ }^{4} \mathrm{~F}\right)$; a segunda ocorre próximo de 440 $\mathrm{nm}$, corresponde a ${ }^{4} \mathrm{~A}_{2 \mathrm{~g}}\left({ }^{4} \mathrm{~F}\right) \rightarrow{ }^{4} \mathrm{~T}_{1 \mathrm{~g}}\left({ }^{4} \mathrm{~F}\right)$; e as duas últimas, menos pronunciadas, ocorrem em torno de 640 e 740 $\mathrm{nm}$, sendo atribuídas às transições ${ }^{4} \mathrm{~A}_{2 \mathrm{~g}}\left({ }^{4} \mathrm{~F}\right) \rightarrow{ }^{4} \mathrm{~T}_{1 \mathrm{~g}}\left({ }^{2} \mathrm{G}\right)$ e ${ }^{4} \mathrm{~A}_{2 \mathrm{~g}}\left({ }^{4} \mathrm{~F}\right) \rightarrow{ }^{2} \mathrm{E}\left({ }^{2} \mathrm{G}\right)$ [28]. Já o íon $\mathrm{Co}^{2+}$ realiza três transições: ${ }^{4} \mathrm{~A}_{2}(\mathrm{~F}) \rightarrow{ }^{4} \mathrm{~T}_{2}(\mathrm{~F}), \quad{ }^{4} \mathrm{~A}_{2}(\mathrm{~F}) \rightarrow{ }^{4} \mathrm{~T}_{1}(\mathrm{~F}) \quad$ e $\quad{ }^{4} \mathrm{~A}_{2}(\mathrm{~F}) \rightarrow{ }^{4} \mathrm{~T}_{1}(\mathrm{P}) \quad$ [29].

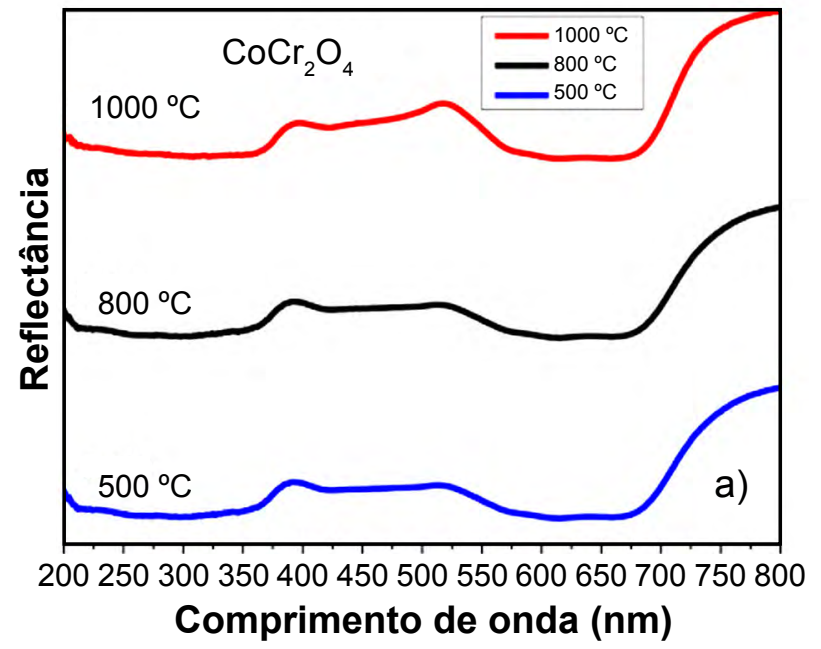

Neste estudo, os resultados condisseram com a literatura, já que, pelos espectros UV-vis, foi possível observar que a cor da cromita $\mathrm{CoCr}_{2} \mathrm{O}_{4}$ foi ocasionada pela sobreposição das bandas das transições do $\mathrm{Co}^{2+} \mathrm{em}$ sítio tetraédrico e das bandas das transições do $\mathrm{Cr}^{3+}$ em sítio octaédrico. Deste modo, as cromitas apresentaram coloração verde. Somando-se a isso, observou-se que as cores dos pigmentos de $\mathrm{Co}_{\mathrm{x}} \mathrm{Zn}_{1-\mathrm{x}} \mathrm{Cr}_{2} \mathrm{O}_{4}$ ficaram mais claras que a amostra $\mathrm{CoCr}_{2} \mathrm{O}_{4}$. Isso foi devido à substituição de íons $\mathrm{Co}^{2+}$ por $\mathrm{Zn}^{2+}$, pois como o $\mathrm{Zn}^{2+}$ não realiza transição d-d (possui $\left.\mathrm{d}^{10}\right)$ não contribui para a cor e, portanto, com a redução da quantidade de íons cromóforos no sistema, é esperado que a intensidade da contribuição do $\mathrm{Co}^{2+}$ seja reduzida, fato este evidenciado pelo aumento do percentual de reflectância $\mathrm{L}$ nos espectros das amostras dopadas.

A Tabela II mostra os valores dos parâmetros CIE-L*a*b* dos pigmentos. Constatou-se que as cores dos pigmentos de $\mathrm{CoCr}_{2} \mathrm{O}_{4}$ obtidos por tratamento nas três temperaturas foram resultantes das contribuições dos deslocamentos

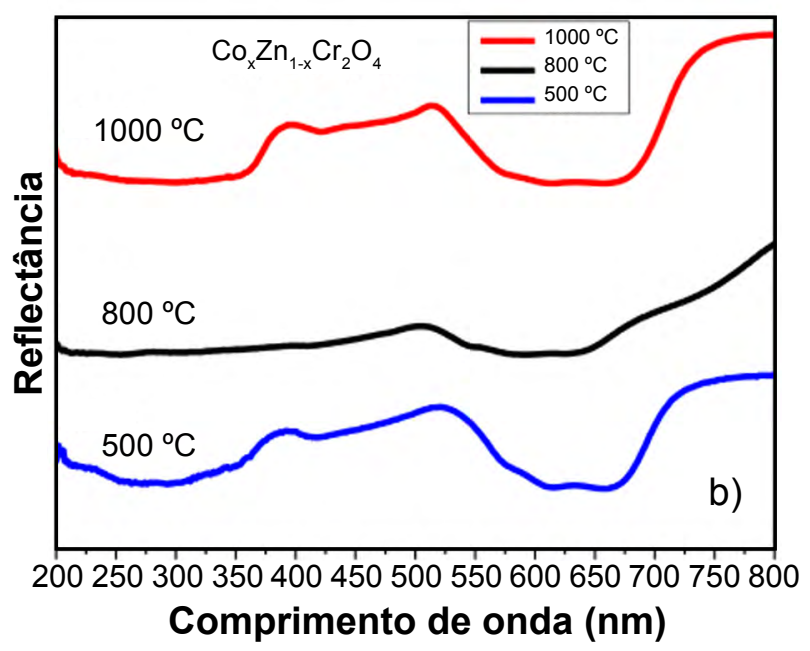

Figura 5: Espectros de reflectância UV-vis de $\mathrm{CoCr}_{2} \mathrm{O}_{4}$ (a) e $\mathrm{Co}_{x} \mathrm{Zn}_{1-x} \mathrm{Cr}_{2} \mathrm{O}_{4}$ (b) tratados a 500, 800 e $1000{ }^{\circ} \mathrm{C}$.

[Figure 5: UV-vis reflectance spectra of $\mathrm{CoCr}_{2} \mathrm{O}_{4}($ a $)$ and $\mathrm{Co}_{x} \mathrm{Zn}_{1-x} \mathrm{Cr}_{2} \mathrm{O}_{4}\left(\right.$ b) heat treated at 500, 800 and $1000{ }^{\circ} \mathrm{C}$.] 
Tabela II - Dados colorimétricos das amostras no sistema CIE-L*a*b*.

[Table II - Colorimetric data of samples in the CIE-L*a*b* system.]

\begin{tabular}{ccccc}
\hline Amostra & Temperatura $\left({ }^{\circ} \mathrm{C}\right)$ & $\mathrm{L}^{*}$ & $\mathrm{a}^{*}$ & $\mathrm{~b}^{*}$ \\
\hline \multirow{3}{*}{$\mathrm{CoCr}_{2} \mathrm{O}_{4}$} & 500 & 43,88 & $-19,84$ & $-8,64$ \\
& 800 & 40,18 & $-9,04$ & $-8,92$ \\
& 1000 & 41,40 & $-9,00$ & $-11,17$ \\
\hline \multirow{2}{*}{$\mathrm{Co}_{\mathrm{x}} \mathrm{Zn}_{1-\mathrm{x}} \mathrm{Cr}_{2} \mathrm{O}_{4}$} & 500 & 48,71 & $-10,75$ & $-1,66$ \\
& 800 & 50,19 & $-8,92$ & 0,06 \\
& 1000 & 53,85 & $-15,97$ & $-1,68$ \\
\hline
\end{tabular}

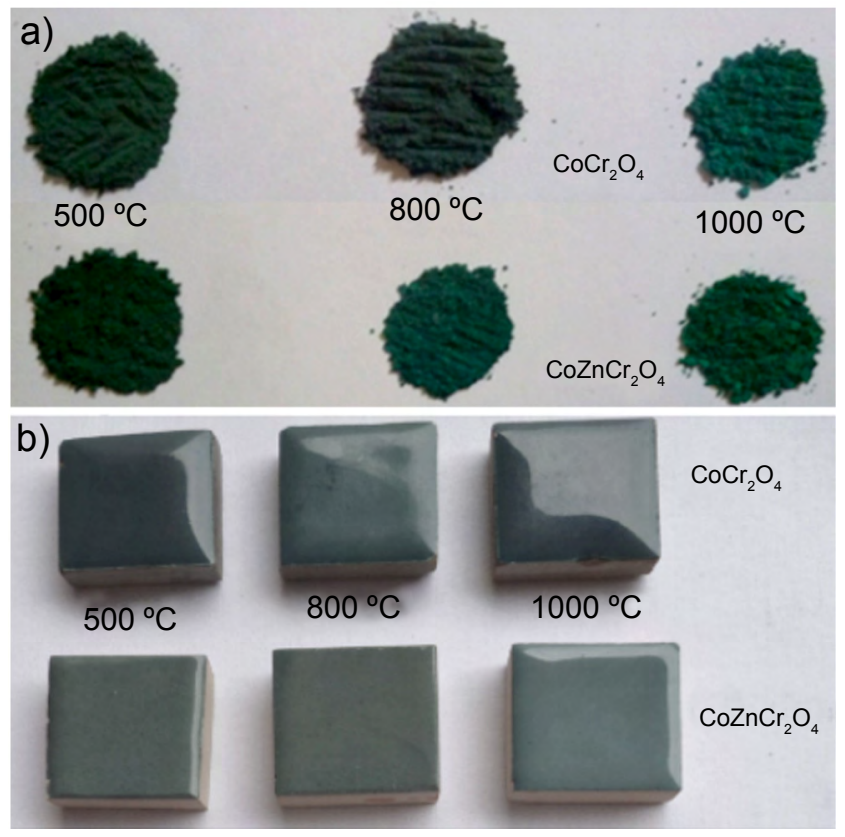

Figura 6: Imagens das amostras de pigmentos (a) e esmaltes aplicados no substrato cerâmico após tratamento térmico a $1100{ }^{\circ} \mathrm{C}(\mathrm{b})$.

[Figure 6: Images of pigment samples (a) and glazes applied on ceramic substrate after heat treatment at $\left.1100{ }^{\circ} \mathrm{C}(\mathrm{b}).\right]$

das coordenadas -a (direção do verde) e -b (direção do azul), resultando em cores em tons de azul esverdeado. Já em relação ao pigmento $\mathrm{Co}_{\mathrm{x}} \mathrm{Zn}_{1-\mathrm{x}} \mathrm{Cr}_{2} \mathrm{O}_{4}$, identificou-se um deslocamento na direção da coordenada -a, enquanto $a$ coordenada $b$ permaneceu praticamente no centro do eixo, resultando em cores finais verdes, atribuídas apenas ao deslocamento da coordenada -a. A análise de cor corroborou os resultados dos espectros de reflectância das amostras (Fig. 5). A Fig. 6 exibe as cores dos pigmentos sintetizados, bem como os esmaltes aplicados no substrato cerâmico. Observou-se que os pigmentos mantiveram suas colorações na temperatura de queima do revestimento $\left(1100{ }^{\circ} \mathrm{C}\right)$, o que sugeriu que houve compatibilidade química entre os componentes do esmalte transparente brilhante e dos pigmentos obtidos. Além disso, verificouse que as aplicações que usaram o pigmento dopado com zinco $\left(\mathrm{Co}_{\mathrm{x}} \mathrm{Zn}_{1-\mathrm{x}} \mathrm{Cr}_{2} \mathrm{O}_{4}\right)$ foram mais claras que os pigmentos sem dopagem $\left(\mathrm{CoCr}_{2} \mathrm{O}_{4}\right)$, conforme foi verificado nos resultados da reflectância (Fig. 5) e da colorimetria dos materiais sintetizados (Tabela II).

\section{CONCLUSÕES}

Os resultados obtidos indicaram que o método gelatina mostrou-se um processo favorável na obtenção de pigmentos cristalinos com estruturas $\mathrm{CoCr}_{2} \mathrm{O}_{4}$ e $\mathrm{Co}_{\mathrm{x}} \mathrm{Zn}_{1-\mathrm{x}} \mathrm{Cr}_{2} \mathrm{O}_{4}$ a baixas temperaturas, sendo um método simples, econômico e produtivo, com potencial de ser viável a sua produção em grande escala. Os difratogramas de raios X apresentaram reflexões da estrutura espinélio com simetria cúbica monofásica na maioria das amostras sintetizadas. As cores dos pigmentos de $\mathrm{CoCr}_{2} \mathrm{O}_{4}$ e $\mathrm{Co}_{\mathrm{x}} \mathrm{Zn}_{1-\mathrm{x}} \mathrm{Cr}_{2} \mathrm{O}_{4}$ foram azuis esverdeados e verdes, respectivamente, conforme determinação das coordenadas do espaço de cores do sistema CIE-L*a*b* e foram resultantes da sobreposição das bandas de reflectância dos íons cromóforos $\mathrm{Cr}^{3+} \mathrm{e} \mathrm{Co}^{2+}$. Os pigmentos apresentaram potencial aplicação em pisos e revestimentos cerâmicos.

\section{AGRADECIMENTOS}

Os autores agradecem à empresa VITTRA Fritas e Esmaltes Cerâmicos, ao LACOM-UFPB e à CAPES pelo suporte financeiro.

\section{REFERÊNCIAS}

[1] L.C. Porto, C.M.Z. Cristiano, V. Soldi, in $9^{\circ}$ Congr. Bras. Polímeros 4 (2007) 17.

[2] Z. Wang, S.K. Saxena, P. Lazor, H.S.C. O'neill, J. Phys. Chem. Solids 64 (2006) 425.

[3] C.S. Xavier, L.E.B. Soledade, E. Longo, C.A. Paskocimas, S.J.G. Lima, I.M.G. Santos, A.G. Souza, in Brazil. MRS Meet. (2006).

[4] A.C. Martin, L.M. Schabbach, M.V. Folgueras, Cerâmica 65, 376 (2019) 562.

[5] F. Bondioli, F. Andreola, L. Barbieri, T. Manfredini, A.M. Ferrari, J. Eur. Ceram. Soc. 27 (2007) 3483.

[6] E. Ozel, S. Turan, J. Eur. Ceram. Soc. 23 (2003) 2097.

[7] R.N. de Almeida, S.F. Santos, J.A. Sampaio, A.B. da Luz, T. Ogasawara, M.C. de Andrade, Cerâmica 53, 325 (2007) 57.

[8] G. George, Dyes Pigments 112 (2015) 81.

[9] R.C. Olegário, E.C.F. Souza, J.F.M. Borges, J.B.M. Cunha, A.V.C. Andrade, S.R.M. Antunes, A.C. Antunes, Dyes Pigments 97 (2013) 113.

[10] A.F. Costa, P.M. Pimentel, D.M.A. Melo, M.S.C. Câmara, L. Chantelle, M.A.F. Melo, R.L.B.A. Medeiros, Cerâmica 62, 362 (2016) 179.

[11] J.M.B. Wanderley, F.M.M. Borges, J.P. Ribeiro, P.M. Pimentel, D.M.A. Melo, Cerâmica 59, 349 (2013) 156.

[12] J.L.S. Dutra, G.C.B. Dantas, P.M. Pimentel, F.M. Aquino, A.F. Costa, A.C. Lima, R.M.P.B. Oliveira, 
Cerâmica 64, 371 (2018) 413.

[13] N.A.S. Nogueira, V.H.S. Utuni, Y.C. Silva, P.K. Kiyohara, I.F. Vasconcelos, M.A.R. Miranda, J.M. Sasaki, Mater. Chem. Phys. 163 (2015) 402.

[14] M. Kakihana, J. Solgel Sci. Technol. 6 (1996) 7. [15] H.M. Rietveld, J. Appl. Crystallogr. 2 (1969) 65.

[16] L. Lutterotti, MAUD v.2.046 (2006) www.ing.unitn. it/wmaud/.

[17] F.S. Oliveira, P.M. Pimentel, R.M.P.B. Oliveira, D.M.A. Melo, M.A.F. Melo, Mater. Lett. 64 (2010) 2700.

[18] S.A. Eliziário, "Pigmentos de estrutura espinélio à base de cromo trivalente", Diss. Mestr, UFPB, João Pessoa (2007).

[19] P.R.G. Gonçalves Jr, J.H.G. Rangel, M.M. Oliveira, E. Azevedo, L.P.S. dos Santos, E. Longo, Cerâmica 52, 324 (2006) 293.

[20] N. Betancur-Granados, O.J. Restrepo-Baena, J. Eur. Ceram. Soc. 37 (2017) 5051.

[21] Z. Tian, C. Zhu, J. Wang, Z. Xia, Y. Liu, S. Yuan, J.
Magn. Magn. Mater. 377 (2015) 176.

[22] M.B.M. Coelho, M.M. Oliveira, I.C. Nogueira, J.H.G. Rangel, J.S. Vasconcelos, E. Azevedo, A.P. Maciel, E. Longo, Cerâmica 64, 369 (2018) 49.

[23] K.T. Jacob, G.M. Kale, G.N.K. Iyengar, J. Mater. Sci. 21, 8 (1986) 2753.

[24] W. Li, H. Cheng, Solid State Sci. 9, 8 (2007) 750.

[25] A.F. Costa, P.M. Pimentel, F.M. Aquino, D.M.A. Melo, M.A.F. Melo, Mater. Sci. Forum 798-799 (2014) 160.

[26] M. Gaudon, A. Apheceixborde, M. Menetrier, A.L. Nestour, A. Demourgues, Inorg. Chem. 48 (2009) 9085.

[27] L.K.C. Souza, J.R. Zamian, G.N. Rocha Filho, L.E.B. Soledade, I.M.G. Santos, A.G. Souza, T. Scheller, R.S. Angelica, C.E.F. Costa, Dyes Pigments 81 (2009) 187.

[28] M. Dondi, G. Cruciani, G. Guarini, F. Matteucci, M. Raimondo, Ceram. Int. 32 (2006) 393.

[29] M. Llusar, A. Fores, J.A. Badenes, J. Calbo, M.A. Tena, G. Monros, J. Eur. Ceram. Soc. 21 (2001) 1121.

(Rec. 09/05/2018, Rev. 23/02/2019, 23/03/2019, 10/05/2019, 09/07/2019, Ac. 27/07/2019) 